\title{
Timescales of crystallization and viscous flow of the bulk glass-forming Zr-Ti-Ni-Cu-Be alloys
}

\author{
Theodore Waniuk, Jan Schroers, ${ }^{*}$ and William L. Johnson \\ California Institute of Technology, Keck Laboratory of Engineering Materials 138-78, Pasadena, California 91125
}

(Received 7 June 2002; published 15 May 2003)

\begin{abstract}
Crystallization behavior and equilibrium viscosity of a series of alloys in the $\mathrm{Zr}$-Ti-Cu-Ni-Be system are studied using multiple techniques to determine the various contributions to glass-forming ability. Lowtemperature time-temperature-transformation diagrams of alloys whose compositions lie at equally spaced points along the tie line from $\mathrm{Zr}_{38.5} \mathrm{Ti}_{16.5} \mathrm{Cu}_{15.25} \mathrm{Ni}_{9.75} \mathrm{Be}_{20}$ to $\mathrm{Zr}_{46.25} \mathrm{Ti}_{8.25} \mathrm{Cu}_{7.5} \mathrm{Ni}_{10} \mathrm{Be}_{27.5}$ are measured during isothermal annealing of initially amorphous specimens. Surprisingly, for all investigated alloys, a primary quasicrystalline phase forms at a rate which varies substantially with alloy composition. Subsequent constant heating measurements, x-ray-diffraction patterns obtained after various states of annealing, beam bending viscosity results, and previous thermal analysis are all used to describe the influences on crystallization in this series. The description of both the kinetic and thermodynamic aspects of crystallization allows for an explanation of the crystallization mechanism. In addition, it explains why, in this series, thermal stability is greatest in those alloys with the poorest glass-forming ability. Overall, the investigations reveal that simple criteria like thermal stability or high viscosity fail to predict the glass-forming ability in complex bulk glass-forming systems.
\end{abstract}

DOI: 10.1103/PhysRevB.67.184203

PACS number(s): 81.10.Aj, 82.30.Lp, 65.60.+a, 66.20.+d

\section{INTRODUCTION}

In the 1960s it was discovered that certain binary metallic systems could be forced to solidify without crystallizing if cooled with a rate of approximately $10^{6} \mathrm{~K} / \mathrm{s} .{ }^{1}$ Subsequent decades witnessed the development of numerous multicomponent alloys with cooling rates to form a glass ranging from 100 to $1 \mathrm{~K} / \mathrm{s}^{2-5}$ allowing such materials to be cast in bulk form, i.e., with dimensions on the order of centimeters. In general, it has been found that glass-forming ability (GFA) in these bulk metallic glasses (BMG's) tends to increase as more components are added to each alloy composition, implying a "confusion principle:" ${ }^{6}$ increasing the components in an alloy system destabilizes competing crystalline phases which form during cooling, effectively frustrating the alloy's ability to crystallize by making the liquid phase more stable relative to the crystalline phases. Over time, the discovery of new and better glass formers has prompted a search for a comprehensive underlying rule for predicting GFA's based on common thermodynamic and kinetic properties of BMG compositions.

From a thermodynamic standpoint, bulk glass formers naturally exhibit characteristics which indicate a low driving force for crystallization in the supercooled liquid. This low driving force causes reduced nucleation rates and therefore correspondingly improved GFA's. Thermal analysis of metallic glasses, particularly the more stable ones, allows determination of the Gibbs free-energy difference $\Delta G_{1-x}$ between the liquid and solid. Generally, it has been found that bulk GFA is correlated with smaller values of $\Delta G_{1-x} .{ }^{7}$

Despite an observed discontinuity in the specific heat at the glass transition, it is incorrect to describe the glass transition as a thermodynamic phase transition. $T_{g}$, the glass transition temperature, depends on the experimental heating or cooling rate used during measurements, and thus kinetic parameters such as viscosity have a significant influence on the GFA of a particular system. Bulk glass formers generally exhibit high equilibrium viscosities over a large temperature range between the melt and the amorphous solid. The temperature dependence of the equilibrium viscosity can be described by a fragility parameter $D^{*}$. Low values of $D^{*}$ are associated with low melt viscosities and large changes in kinetic properties near $T_{g}$ (fragile liquids), while high $D^{*}$ values correspond to high melt viscosities and small changes in kinetic properties (strong liquids). ${ }^{8} \mathrm{BMG}$ alloys, with melt viscosities substantially higher than pure metals, fall into the latter category. ${ }^{9}$ Such behavior has a distinct influence on crystallization by retarding the formation and growth of crystalline nuclei during cooling, resulting in better GFA overall.

To describe and predict the ability to bypass crystallization in undercooled alloys, Turnbull suggested a criterion which takes into account thermodynamic and kinetic factors involved in nucleation and growth. He demonstrated that as $T_{r g}$, the glass transition temperature $T_{g}$ divided by the liquidus temperature $T_{1}$, approached $\frac{2}{3}$, homogeneous nucleation in an alloy would be substantially suppressed, leading to BMG behavior. $T_{r g}$ has been used to predict the GFA of a large number of glass-forming compositions. However, among the more complex multicomponent bulk metallic glasses have been found several "rogue" glasses with glassforming abilities that do not fit the predictions of the Turnbull criterion. ${ }^{10}$ For example, despite high experimentally determined values of $T_{r g}$ for $\mathrm{Zr}_{41.2} \mathrm{Ti}_{13.8} \mathrm{Cu}_{12.5} \mathrm{Ni}_{10} \mathrm{Be}_{22.5}$, microstructural investigations have revealed a high density of nanocrystals after annealing at low temperatures in the vicinity of the glass transition in $\mathrm{Zr}_{41.2} \mathrm{Ti}_{13.8} \mathrm{Cu}_{12.5} \mathrm{Ni}_{10} \mathrm{Be}_{22.5} .{ }^{12}$ This implies a high nucleation rate, contradicting predictions based on $T_{r g}$. Multiple explanations have been posited to explain this behavior, including phase separation in the undercooled liquid, ${ }^{12}$ a linked-flux model of nucleation, ${ }^{13}$ and catastrophic nucleation. ${ }^{14}$

Recently, studies have been performed on a series of alloys spaced equally in composition from $\mathrm{Zr}_{41.2} \mathrm{Ti}_{13.8} \mathrm{Cu}_{12.5} \mathrm{Ni}_{10} \mathrm{Be}_{22.5}$ (Vit1) to 
$\mathrm{Zr}_{46.25} \mathrm{Ti}_{8.25} \mathrm{Cu}_{75} \mathrm{Ni}_{10} \mathrm{Be}_{27.5}$ (Vit4). ${ }^{15}$ These alloys are distinguished from each other by their critical cooling rate $R_{c}$ and thermal stability, represented by $\Delta T$, the temperature interval between the glass transition and the onset of crystallization. In the past, both of these parameters have been commonly and interchangeably used to describe the relative GFA for a large number of metallic glass compositions from multiple alloy families. For the Vit1-Vit4 series described above, however, the thermal stability tends to decrease as the critical cooling rate decreases (i.e., the GFA increases). This apparent contradiction makes $\mathrm{Zr}$ - $\mathrm{Ti}-\mathrm{Cu}-\mathrm{Ni}$-Be a favorable system to elucidate the various influences on GFA.

The present paper will discuss the results of crystallization kinetics and flow behavior studies performed on seven alloys along the tie line in composition space between Vit1 and Vit4. The various influences on GFA will be examined together as a function of composition: thermal stability, timescales for crystallization, timescales for viscous flow, thermodynamic properties, and previous results concerning nucleation density will all be utilized. Crystallization studies will be summarized in the form of time-temperaturetransformation (TTT) diagrams, and viscosity data obtained using beam bending methods will be presented for all alloys and discussed in the framework of the fragility concept. These data show that even though the alloys near Vit4 exhibit the slowest crystallization kinetics upon heating amorphous material, they also have the lowest equilibrium viscosities for the same temperature range, counter to expectations. To assist in describing the crystallization pathways in this system, x-ray-diffraction (XRD) measurements taken after isothermal anneals of varying duration will also be presented. These XRD data reveal that all the alloys form a metastable quasicrystalline primary phase prior to subsequent bulk crystallization events, and thus the combined XRD studies and thermal analysis results facilitate an explanation of the crystallization process in this alloy series.

\section{EXPERIMENTAL PROCEDURE}

Samples of amorphous $\mathrm{Zr}_{38.5} \mathrm{Ti}_{16.5} \mathrm{Cu}_{15.25} \mathrm{Ni}_{9.75} \mathrm{Be}_{20}$ [Vit1(-b)], $\mathrm{Zr}_{39.88} \mathrm{Ti}_{15.12} \mathrm{Cu}_{13.77} \mathrm{Ni}_{9.98} \mathrm{Be}_{21.25}$ [Vit1(-a)], Vit1, $\mathrm{Zr}_{42.63} \mathrm{Ti}_{12.37} \mathrm{Cu}_{11.25} \mathrm{Ni}_{10} \mathrm{Be}_{23.75}$ (Vit1a), $\mathrm{Zr}_{44} \mathrm{Ti}_{11} \mathrm{Cu}_{10} \mathrm{Ni}_{10} \mathrm{Be}_{25}$ (Vit1b), $\mathrm{Zr}_{45.38} \mathrm{Ti}_{9.62} \mathrm{Cu}_{8.75} \mathrm{Ni}_{10} \mathrm{Be}_{26.25}$ (Vit1 c), and Vit4 were prepared with elements of $99.5 \%$ to $99.995 \%$ purity. The tie line used to describe this alloy system can be represented by $\left(\mathrm{Zr}_{37.5+2.5 x} \mathrm{Ti}_{62.5-2.5 x}\right)_{55}\left(\mathrm{Ni}_{3 x} \mathrm{Cu}_{100-3 x}\right)_{41.25-1.25 x} \mathrm{Be}_{3.75+1.25 x}$, where $x=0-25$. After arc melting the constituents in a titanium-gettered argon atmosphere, each ingot was sealed in a quartz tube under a vacuum of $10^{-6}$ mbar, heated above the liquidus for $5 \mathrm{~min}$, and subsequently quenched in water. Isothermal differential scanning calorimetry (DSC) measurements were performed using a Perkin Elmer DSC 7 analyzer. Viscosity measurements were carried out with a Perkin Elmer TMA 7 using a three-point beam bending technique, the details of which are described elsewhere. ${ }^{9}$ Finally, a Co- $K \alpha$ source and an INEL CPS- 120 position sensitive detector were used to acquire XRD data for the various alloys.

Isothermal crystallization studies were performed on material that was heated from room temperature to the anneal-

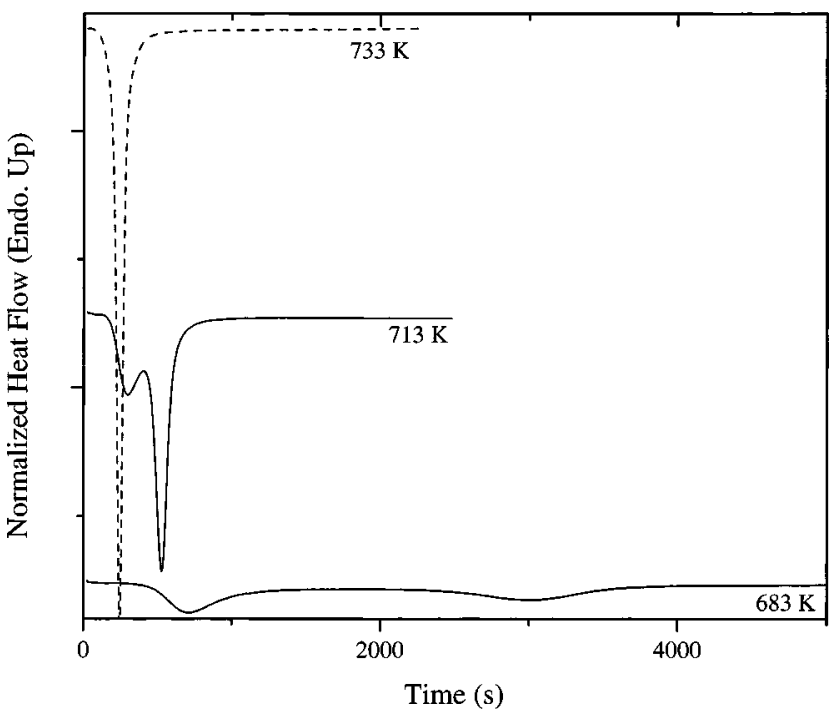

FIG. 1. DSC thermograms of isothermal crystallization of Vit1a at 683,713 , and $733 \mathrm{~K}$.

ing temperature at a rate of $3.33 \mathrm{~K} / \mathrm{s}$. After holding samples at the annealing temperature for a specified length of time, they were cooled to room temperature at $3.33 \mathrm{~K} / \mathrm{s}$. In order to determine the volume fraction crystallized during the isothermal step, subsequent DSC measurements using a constant heating rate of $0.66 \mathrm{~K} / \mathrm{s}$ were also performed on some of the samples.

\section{RESULTS}

Figure 1 shows representative isothermal DSC thermograms of Vit1a. The lowest temperature measurement, taken at $683 \mathrm{~K}$, reveals two sluggish exothermic crystallization events whose peaks are separated by approximately $2300 \mathrm{~s}$. As the annealing temperature is increased to $713 \mathrm{~K}$ [Fig. 1(b)], the spacing in time between successive crystallization events decreases and each crystallization event itself occurs more rapidly, until only one rapid crystallization event is apparent [Fig. 1(c)] at high temperatures.

Summaries of the isothermal crystallization studies are shown in Fig. 2 as time-temperature-transformation (TTT) diagrams. For a given temperature, each TTT diagram shows the time to reach the onset $(\square)$ and end ( $\square$ ) of the first crystallization event and the time to reach the onset $(O)$ and end (-) of the second crystallization event for each alloy. Two general trends are immediately apparent. First, when annealed at high temperatures, most of the alloys crystallize in one rapid event, but all the alloys crystallize in two separate events when annealed at lower temperatures. Second, isothermal stability (the time to reach crystallization) at temperatures near $T_{g}$ tends to increase as the tie line is traversed from Vit1(-b) to Vit4. As shown in Fig. 1 for Vit1a, the first crystallization event for all the alloys ceases to be apparent above a certain temperature; with increasing annealing temperature, the second crystallization event steadily increases in magnitude and rate, eventually dominating the crystallization of the alloy. The temperature at which the crystallization behavior changes varies from $663 \mathrm{~K}$ in Vit4 to $733 \mathrm{~K}$ in Vit1, 

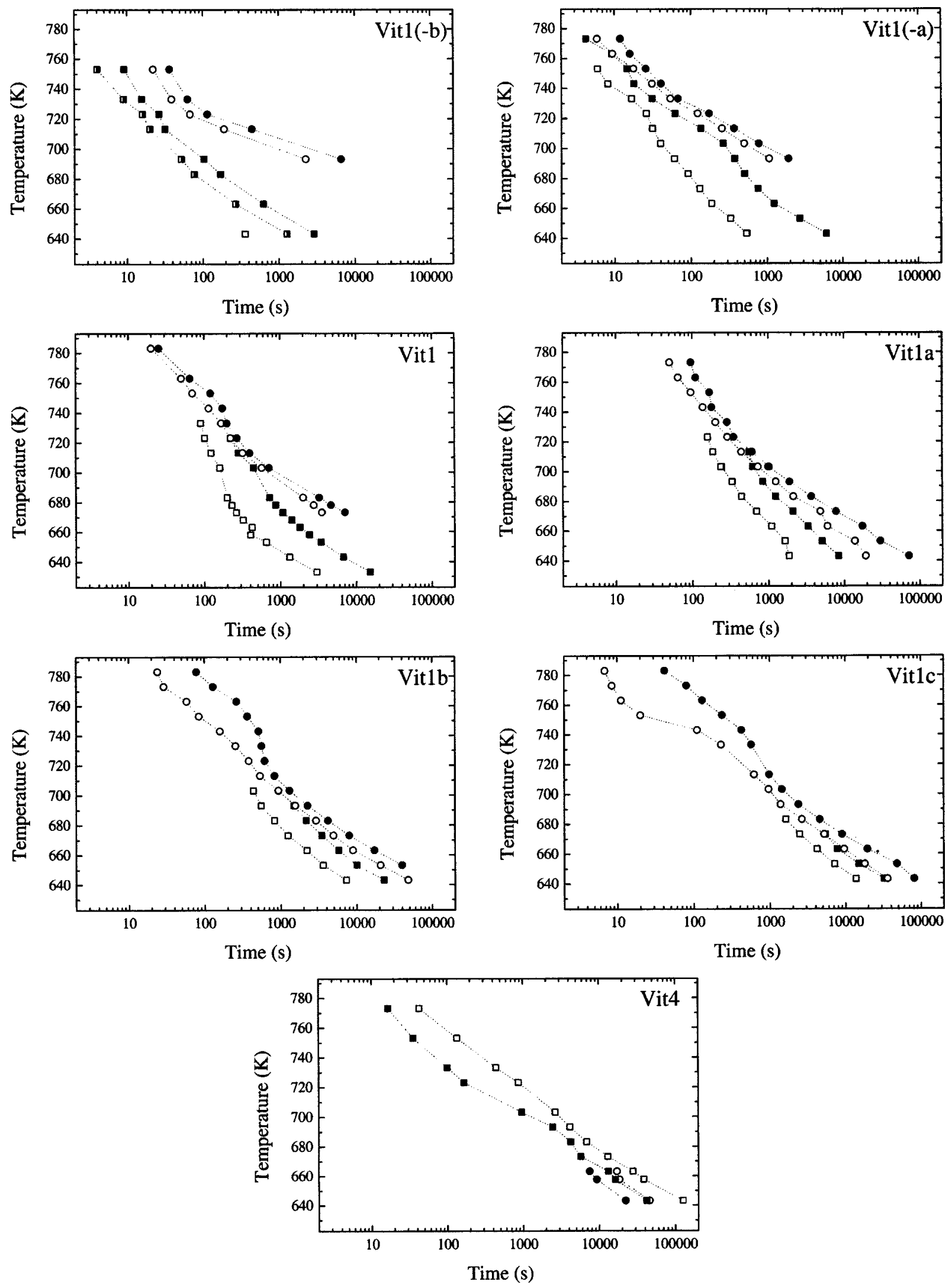

FIG. 2. Time-temperature-transformation (TTT) diagrams measured after heating from the amorphous state for the Vit1(-b)-Vit4 series. The onset $(\square)$ and end $(\boldsymbol{\square})$ of the first crystallization event and the onset $(\bigcirc)$ and end $(\boldsymbol{O})$ of the second crystallization event are shown. For Vit1(-b), the time to the first peak in the heat flow signal, rather than the onset, is shown as half filled squares. Above a certain temperature for Vit1-Vit4, the primary crystallization does not occur, and only one crystallization event is apparent. 


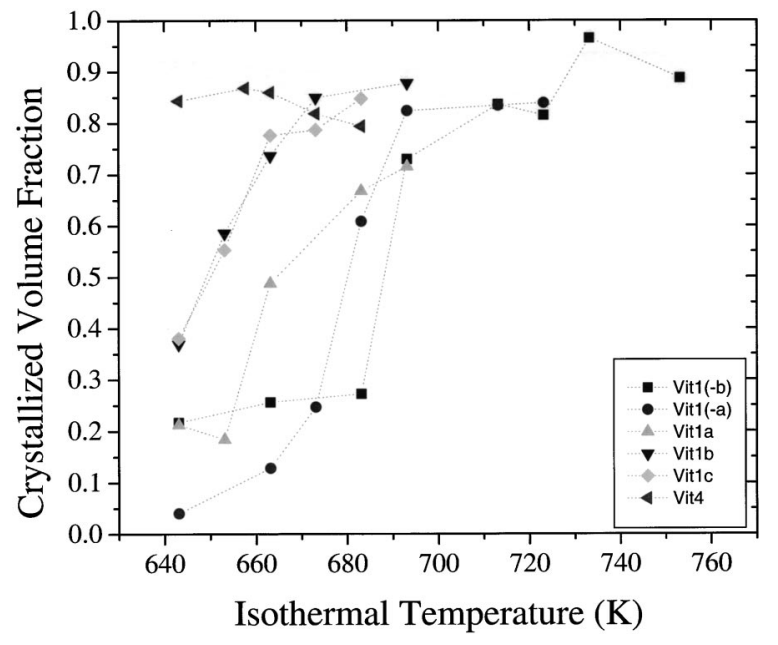

FIG. 3. Crystallized volume fraction vs isothermal annealing temperature for Vit1(-b)-Vit4.

while two crystallization events are always evident for Vit1(-a) and Vit1(-b). In addition to the transition from one to two crystallization events, there is also a dramatic extension of the onset of the first crystallization event to longer times between 673 and $643 \mathrm{~K}$ for each successive alloy in the series. This increase in isothermal stability is particularly noticeable in Vit1b, Vit1c, and Vit4, with the latter requiring annealing times on the order of $5 \mathrm{~h}$ at $643 \mathrm{~K}$ before crystallization sets in. It should be noted that for temperatures below $643 \mathrm{~K}$ long duration heat releases due to crystallization are too small in magnitude to enable accurate determination of onset and end times from DSC data for all the studied alloys. Also, because at high temperatures there is an asymmetry in crystallization behavior during heating versus during cooling, it was not possible to acquire TTT data above the effective "nose" of the diagram for each alloy using isothermal annealing. The DSC used is limited to a maximum heating rate of $3.33 \mathrm{~K} / \mathrm{s}$, and it has been shown that a heating rate of approximately $200 \mathrm{~K} / \mathrm{s}$ is necessary to avoid crystallization upon heating. ${ }^{16}$

Following the isothermal anneals, constant heating rate measurements were performed on the samples in order to determine whether they were completely crystalline. Surprisingly, all the alloys showed some remaining amorphous fraction after isothermal annealing, regardless of annealing temperature. By comparing the heat release during crystallization in these subsequent constant heating rate thermograms with the total heat release detected during constant heating of the as-cast material, the relative volume fraction crystallized during each step was determined and the amount of amorphous material remaining after the isothermal step was thus estimated. The results of these measurements, shown in Fig. 3, reveal a steady increase in volume fraction crystallized during the isothermal step as annealing temperature is increased for all the alloys except Vit4. The latter shows a crystallized volume fraction which remains fairly constant with annealing temperature. To investigate compositional changes in the amorphous material remaining after isothermal annealing, $T_{g}$ was determined for each alloy as well. Figure 4 shows $T_{g}$ onset values derived from constant

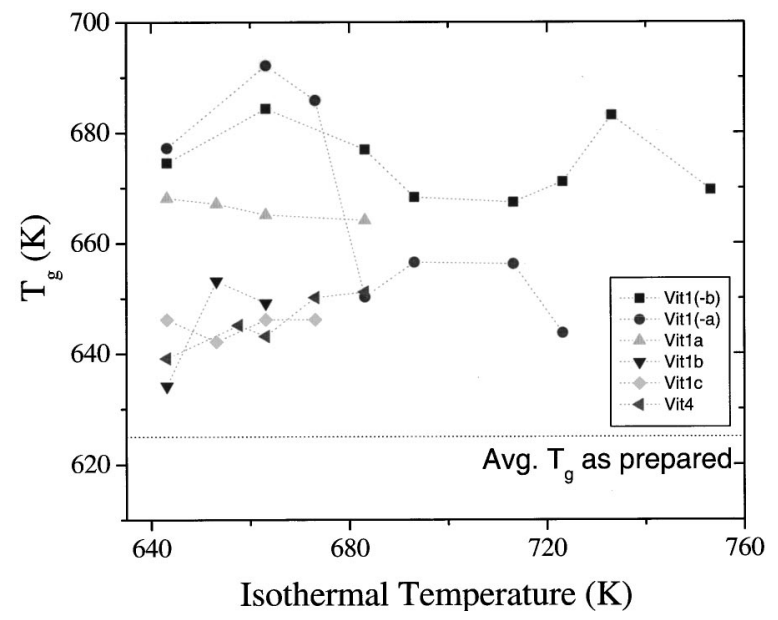

FIG. 4. Glass transition temperature $T_{g}$ of annealed and partially crystallized samples vs isothermal annealing temperature. The horizontal dotted line represents the average $T_{g}$ measured from as-cast samples; $T_{g}$ varies very little with composition in as-cast samples from this series. ${ }^{15}$ Because each sample is already partially crystallized before the constant heating rate measurement used to measure $T_{g}$, the endothermic heat-flow signal during the glass transition is smaller in magnitude and it is more difficult to determine the onset, resulting in the substantial scatter shown.

heating rate measurements of samples processed at different isothermal annealing temperatures. Though considerable scatter is evident for all the alloys, it is clear that $T_{g}$, measured subsequent to isothermal anneals on partially crystallized samples, increases substantially compared to the initially fully amorphous material.

In order to gain some insight into the nature of the phases formed during isothermal annealing, x-ray scans were performed on samples of Vit1-Vit4. These scans are shown in Figs. 5(a)-(e). Prior to the XRD measurements, samples of each alloy were held at higher annealing temperatures where only one crystallization event exists (see Fig. 2) and were quenched to room temperature after the exothermic event was complete. In addition, samples of each alloy were also annealed at low temperatures where two crystallization peaks are evident and were quenched after the first peak and after the second peak. For these, XRD data were gathered immediately after the primary crystallization and also after both primary and secondary crystallization peaks were finished. After primary crystallization during annealing at $703 \mathrm{~K}$ for Vit1, $693 \mathrm{~K}$ for Vit1a, $683 \mathrm{~K}$ for Vit1b, $663 \mathrm{~K}$ for Vit1c, and $633 \mathrm{~K}$ for Vit4, all the alloys show a pattern containing three peaks at approximate $d$ spacings of $2.4,2.3$, and $1.4 \AA$ ( $2 \theta$ $\approx 43^{\circ}, 45^{\circ}$, and $77^{\circ}$ ), indicating the presence of a quasicrystalline phase. This phase has been observed previously in Vit4. ${ }^{17}$ After both crystallization events have finished during low temperature annealing of the three alloys, XRD data exhibit a large number of peaks which result from the presence of $\mathrm{Be}_{2} \mathrm{Zr}, \mathrm{Zr}_{2} \mathrm{Cu}$, and other phases, as has been shown in previous studies. ${ }^{18}$ Though peaks at positions corresponding to the quasicrystalline phase are still present as well, it cannot be unambiguously determined whether the quasicrystalline phase remains after the second crystallization event. There is a difference between the alloys in the types of 

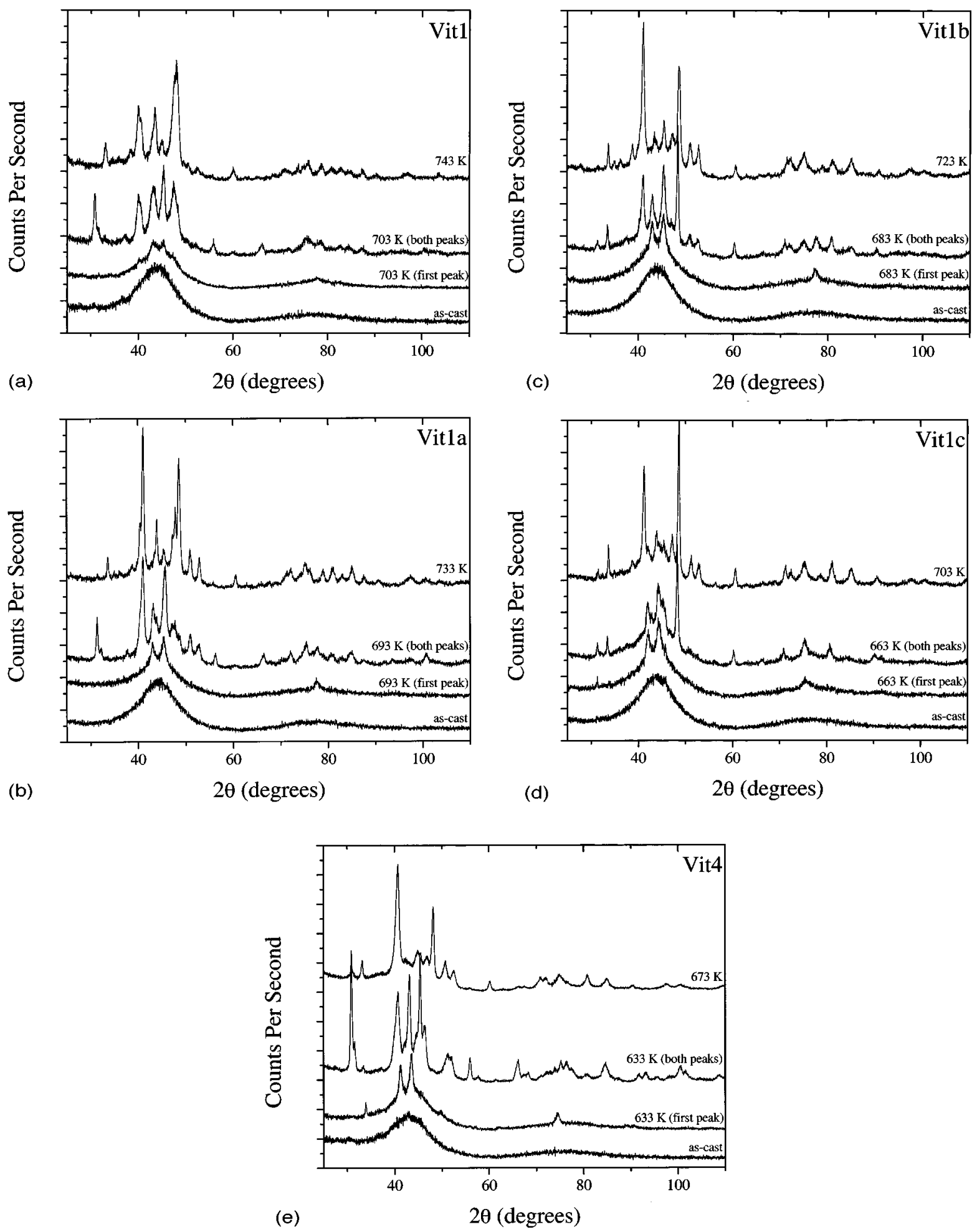

FIG. 5. XRD spectra taken on as-cast material and also after isothermal anneals at various temperatures: (a) Vit1, (b) Vit1a, (c) Vit1b, (d) Vit1c, (e) Vit4. For the low-temperature annealed samples of each alloy, XRD measurements were performed immediately after the first crystallization peak and also immediately after the second peak detected by DSC. XRD measurements were also performed immediately after the single-crystallization event observed during high-temperature annealing.

phases that form after the second crystallization event, however. For example, whereas the peaks at $d=2.6 \AA, d$ $=2.1 \AA$, and $d=2.0 \AA$ are present in both Vit1a and Vit1b, they are absent or exist only as a shoulder in Vit1c. In addi- tion, the peaks at $d=3.1 \AA$ and $d=1.8 \AA$ are present in Vit1b and Vit1c but not in Vit1a. Thus there must be at least two additional phases forming during the second crystallization event, and both of these phases are not necessarily 


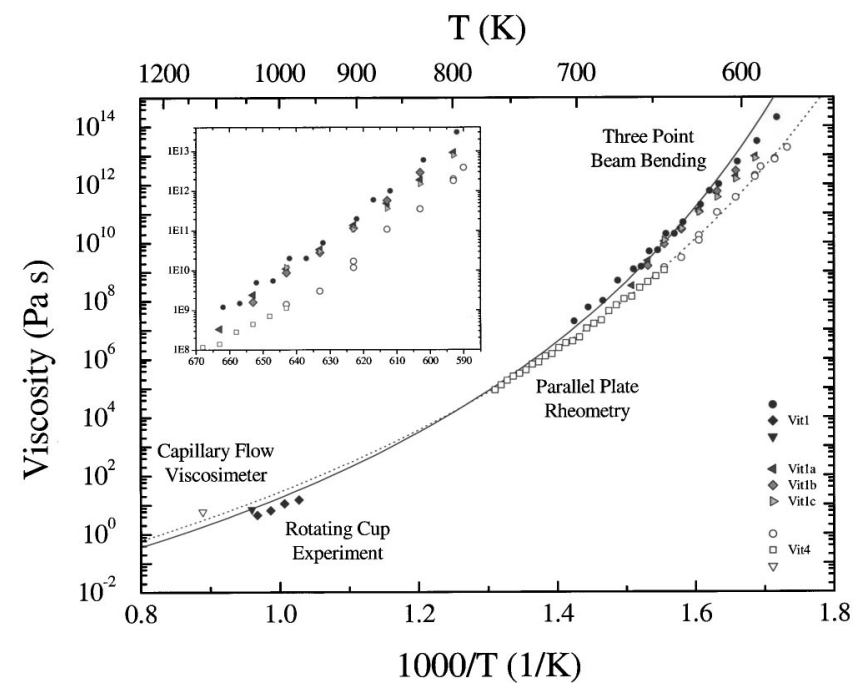

FIG. 6. A summary of the results of equilibrium viscosity measurements for Vit1-Vit4. The three-point beam bending technique was used to acquire data for Vit1a, Vit1b, and Vit1c, and previous results using the same technique as well as additional methods are also shown for Vit1 and Vit4 (see Refs. 9, 21, and 27).

present in each alloy. In contrast, the x-ray measurements taken at high temperatures, where only one crystallization peak is evident, show similar phases for all three alloys, with the $\mathrm{x}$-ray peaks differing mainly in intensity.

Figure 6 depicts data obtained from three-point beam bending viscosity measurements of Vit1, ${ }^{9}$ Vit1a, Vit1b, Vit1c, and Vit4. ${ }^{19}$ From these data, it appears that the equilibrium viscosities of Vit1a, Vit1b, and Vit1c lie between those of Vit1 and Vit4 in the low-temperature regime. In addition, it appears that Vit1c exhibits lower equilibrium viscosity values than the other two alloys in the temperature range studied.

\section{DISCUSSION}

The isothermal crystallization studies reveal the lower part of the TTT diagram for each of the investigated alloys, and a clear trend in crystallization kinetics can be discerned from Fig. 2. Following the tie line in composition space from Vit1(-b)-Vit4, one observes that the primary phase forms fairly early ( $<1000 \mathrm{~s}$ between 643 and $773 \mathrm{~K})$ for Vit1(-b), Vit1(-a), and Vit1. Thus, relative to the rest of the series, these three alloys exhibit decreased resistance to crystallization and therefore decreased isothermal stability. A related result was observed in previous measurements performed on a differential thermal analyzer (DTA) with a constant heating rate: $\Delta T$ was smallest for Vit1(-b)-Vit1 and reached its maximum value in Vit1c. ${ }^{15}$ For all the alloys, crystallization occurs in several steps. Following the primary crystallization event, a substantial slowdown in kinetics is observed, and the second crystallization event occurs at a much later time. In addition, the secondary event appears to dominate the crystallization of the entire sample above some temperature for each alloy, implying a competition between the primary phase and the phases formed during the second event.

The DSC results, combined with XRD data, show that crystallization proceeds through multiple pathways which depend on annealing temperature. At low temperatures, all the alloys first form a quasicrystalline phase. It has been postulated that this metastable phase eventually transforms to one or more new phases after a sufficient amount of annealing time at a given temperature. ${ }^{17,20}$ After the primary crystallization, a second crystallization process produces the remainder of the peaks observed in XRD. Whereas at low temperatures the phases that appear after the formation of the quasicrystals seem to vary somewhat between the alloys, at high temperatures all the alloys appear to crystallize very rapidly, forming a similar distribution of phases. Differences in samples crystallized at low temperature might thus be attributed to differing rates of development of the various phases and possibly also to the appearance and transformation of metastable phases during annealing. At a sufficiently high temperature which varies for each alloy, the quasicrystalline phase cannot form. Each alloy crystallizes in one rapid event above this temperature, and the consistency of the high-temperature XRD results from all the alloys means that they probably represent an equilibrium distribution of crystalline phases common to the $\mathrm{Zr}-\mathrm{Ti}-\mathrm{Cu}-\mathrm{Ni}-\mathrm{Be}$ system.

Because both the nucleation rate and the growth rate of crystalline phases strongly depend on the kinetics in a material, it is important to examine the extent to which viscosity plays a role in the crystallization behavior of the alloy series. As shown in Fig. 6, the three intermediate alloys have similar equilibrium viscosities between 590 and $655 \mathrm{~K}$, and Vit1c exhibits the second lowest equilibrium viscosity in this temperature range. Fitting the viscosity data using the VogelFulcher-Tamman (VFT) relation

$$
\eta(T)=\eta_{0} \cdot \exp \frac{D^{*} \cdot T_{0}}{\left(T-T_{0}\right)}
$$

yields $D^{*}$ values of $21.3,18.9$, and 25.9 for Vita, Vit1b, and Vit1c, respectively. Previous VFT fits have determined $D^{*}$ values of 18.5 for Vit1 (Ref. 9) and 22.7 for Vit4. ${ }^{21}$ The error in $D^{*}$ due to the fitting process for the three middle alloys is approximately \pm 1.3 , though the small number of points combined with the lack of high-temperature data for these alloys increases the overall error relative to previous fits of Vit1 and Vit4. From the viscosity behavior alone, it appears that Vit4 should demonstrate the highest crystallization rate, and its thermal stability should therefore be greatly reduced. However, both Vit4 and Vit1c show the longest times until the first crystallization event within the temperature range of $643-713 \mathrm{~K}$.

When cooled slowly from the melt, most of these alloys typically crystallize at a temperature substantially higher than the highest temperature measured in Fig. 2. The hightemperature crystallization behavior (above the isothermal annealing temperature at which crystallization is most rapid) for this alloy series has also been explored previously. ${ }^{15}$ In this case, crystallization upon cooling at a constant rate resulted in the determination of the critical cooling rate $R_{c}$, and measurements revealed that $R_{c}$ is lowest for Vit1(-b)Vit1, i.e., these alloys are the best glass formers in the series. 


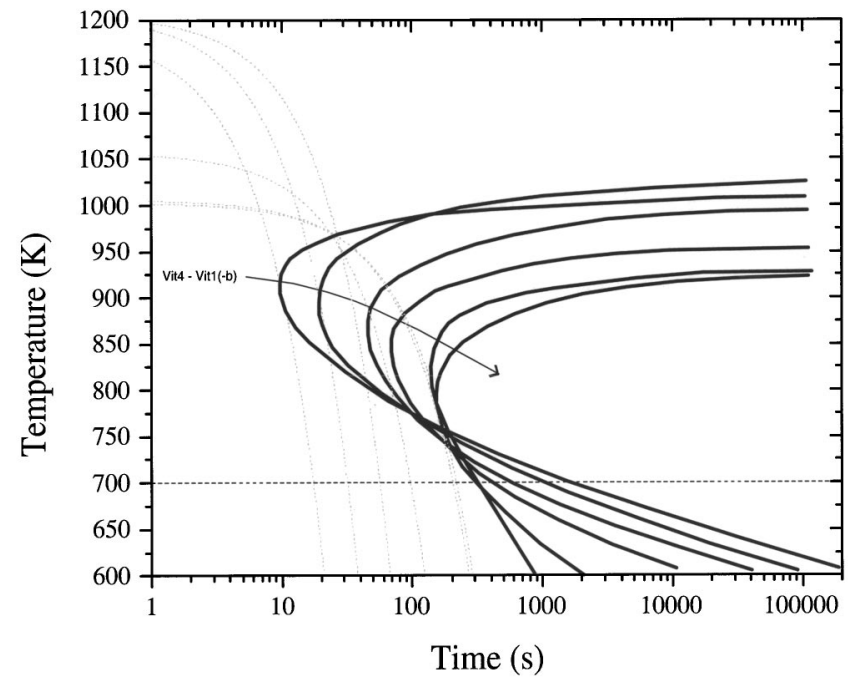

FIG. 7. Estimates of the TTT diagrams for Vit1(-b)-Vit4, based on the results of the present study combined with previous DTA measurements. Upon traversing the series from Vit1(-b)-Vit4, there is a steady extension of the nose to shorter times while at the same time there is an increase in the time to crystallization at low temperatures.

$R_{c}$ steadily increases, however, from Vit1a to Vit4, reaching a maximum of approximately $27 \mathrm{~K} / \mathrm{s}$ in the latter.

Given the results of crystallization experiments both above and below the "nose" of the TTT diagram, some observations can be made about the dominant trends in this alloy series. Though Vit1 represents one of the best glass formers, its thermal stability is substantially reduced compared to Vit1a-Vit4. Conversely, Vit4 exhibits the best thermal stability at low temperatures, yet its glass-forming ability (GFA) is greatly reduced. In order to explain this apparent contradiction, an estimate of the complete TTT diagram for each of the alloys has been made, taking into account the results from Fig. 2 and previous measurements of $R_{c}$. This is shown in Fig. 7. In this diagram, an attempt has been made to show the interplay between GFA, represented by the shortest time to crystallization in the upper part of the TTT diagram, and thermal stability, represented by the time to crystallization at low temperatures near the glass transition. As can be seen in Fig. 7, Vit1(-b)-Vit1 all have low $R_{c}$ values, resulting in a TTT nose which is extended to longer times (on the order of $100 \mathrm{~s}$ ). The low-temperature portion of the TTT diagram varies between these alloys, however, with the onset of crystallization pushed to earlier times in Vit1(-b) and Vit1(-a), indicating substantially reduced thermal stability. From Vitla-Vit4, there is a consistent evolution in the shape of the TTT diagram. The nose for each subsequent alloy begins at earlier times, mirroring the increase in $R_{c}$, while at the same time the onset of crystallization for temperatures below the nose extends to longer times as the thermal stability increases. It is obvious from this diagram that a low critical cooling rate does not necessarily imply a high thermal stability in bulk glass-forming compositions; completely different crystallization mechanisms operate in the high- and low-temperature regimes.

For most of the series, it has been found that there exists a decomposition process in the liquid prior to crystallization. It is possible that the incubation time preceding quasicrystal formation at low temperatures is directly influenced by such a process. As it has been suggested in several investigations that decomposition is the rate limiting step for crystallization, $^{22,23}$ increases in isothermal stability from Vit1(-b) to Vit4 could therefore be attributed to differences in the timescale for decomposition for each alloy. Löffler et al. demonstrated the existence of spatially correlated inhomogeneities in Vit1-Vit4 after annealing at various temperatures. ${ }^{24}$ Critical temperatures, below which decomposition occurs, were reported to be 670,649 , and $642 \mathrm{~K}$ for Vit1, Vit1a, and Vit1b respectively. In addition, an interference maximum resulting from the existence of spatially correlated inhomogeneities was found only at $623 \mathrm{~K}$ for Vit1c, and none was observed for Vit4 in the temperature range studied. The range of critical temperatures indicates that decomposition at a given annealing temperature near $T_{g}$ occurs most rapidly in Vit1, slows down steadily as the tie line is traversed, and is negligibly slow in Vit4. Consequently, the decomposition proceeds fastest in the alloys near Vit1, causing local composition shifts throughout the liquid and reducing the barrier to crystallization in those regions where the composition shifts toward that of the primary phase. Though Vit1 has the most sluggish kinetics of the studied alloys, its resistance to crystallization may thus be mitigated by such composition fluctuations. Vit1c and Vit4, on the other hand, may show such dramatic increases in isothermal stability because the timescale for decomposition has been substantially increased despite their more rapid kinetics. Overall, the scenario described above predicts a crystallization process which is limited at low temperature by the rate and extent of decomposition; high-temperature crystallization in these alloys, represented by the varying critical cooling rate, is not influenced by such a process.

If a decomposition process influences the formation of primary quasicrystals in this alloy series, a correlation should be observable between $T_{t r}$, the temperature above which crystallization ceases to occur in two steps (i.e., no quasicrystallization occurs), and $T_{c}$, the critical temperature cited above. From Fig. 2, the former temperatures are 733, 723, 703,683 , and $663 \mathrm{~K}$ for Vit1-Vit4, while the latter temperatures are 670, 649, 642, and $623 \mathrm{~K}$ for the first four alloys. Clearly, quasicrystals still form during isothermal annealing at temperatures well above the cited values of $T_{c}$ for all the alloys. Despite the lack of agreement between these temperature values, however, it is still possible that the decomposition mechanism has some effect on crystallization above $T_{c}$. The previous small-angle neutron-scattering SANS results reveal correlated inhomogeneities in each alloy composition below its respective $T_{c}$ due to the formation of nanocrystals in phase separated domains with favorable composition. In other words, these values of $T_{c}$ represent an intersection of the alloy composition with a coherent spinodal, below which the alloy is unstable with respect to composition fluctuations and forms regularly spaced decomposed domains. At temperatures higher than the coherent spinodal but below the coherent miscibility gap, the liquid is in a metastable state, and decomposition into two chemically different under- 
cooled liquids must overcome a nucleation barrier. In this temperature regime, decomposed regions that form will be uncorrelated in space, as will be the quasicrystals which form in those regions. Such a distribution would not be revealed by SANS. At $T_{t r}$, each alloy's crystallization behavior changes abruptly because the barrier to nucleation of a phase separated domain in the undercooled liquid becomes comparable to the barrier to nucleation of the competing hightemperature phase. Thus above $T_{t r}$, the primary phase which forms precludes the formation of quasicrystals and triggers a rapid series of crystallization events which manifest as one large heat release in DSC thermograms.

The preceding discussion of the variation in crystallization kinetics among the alloys and its relation to changing decomposition kinetics has not taken into account some aspects of the polymorphic nature of the crystallization in these alloys. In order to explain how a composition fluctuation in the undercooled liquid affects crystallization, not only must the kinetics of the decomposition process be considered but also the amplitude of the composition fluctuations, where amplitude refers to the composition difference between the nominal material and the primary phase. Quasicrystals are known to have a narrow composition (meta)stability range. At low undercoolings, the growth rate controls crystallization in Zr-based alloys, ${ }^{25}$ and it has been found that the growth rate for quasicrystalline phases depends on the chemical short-range order and not the topological shortrange order, as the nucleation rate does. ${ }^{26}$ The alloy with the largest composition difference between the primary quasicrystalline phase and the nominal composition should have the most sluggish crystallization kinetics. Considering this, the composition difference between the nominal glass and the primary phase might also be a crucial factor influencing the thermal stability.

Based on the experimental results detailed above, the early stages of crystallization at temperatures far below the TTT nose of each alloy in this series proceed in the following manner. After a period of time required to create a composition fluctuation in which a nucleus forms, a quasicrystalline phase appears in the undercooled liquid. This transition is nonpolymorphic, and the remaining amorphous matrix has an altered composition following primary crystallization. The volume fraction that crystallizes as a quasicrystalline phase lies between $4 \%$ and $96 \%$ for the compositions and temperature ranges studied. The new matrix composition shows, on the average, an increased $T_{g}$, as revealed by constant heating rate DSC thermograms taken subsequent to the isothermal anneals. This higher $T_{g}$ results from an increase in liquid density which slows down the crystallization kinetics, and the primary crystallization therefore stabilizes the glass. However, this does not necessarily mean that the GFA or thermal stability of the new composition is superior to the original composition. The thermal stability, in fact, is lower for the material containing quasicrystals, because the matrix crystallizes at a lower temperature during subsequent heating; after isothermal annealing between 643 and $653 \mathrm{~K}$, Vit1a, Vit1b, Vit1c, and Vit4 show average decreases in $\Delta T$ of $61,58,54$, and $50 \mathrm{~K}$, respectively, compared to the as-cast material. Such a shift in crystallization temperature might be caused by the composition of the matrix shifting closer to that of a competing crystalline phase.

\section{CONCLUSION}

The crystallization behavior of the Vit1(-b) - Vit4 series was studied using multiple techniques in order to determine and compare the various contributions to GFA. Isothermal annealing experiments were performed on samples heated from the amorphous solid into the supercooled liquid region, and constant heating experiments were conducted subsequent to isothermal anneals. In addition, XRD scans were taken on samples annealed at multiple temperatures and for various durations. To study the kinetics, beam bending viscosity measurements were used to determine the temperature dependence of the equilibrium viscosity of Vit1a-Vit1c. The results show that all the alloys form a quasicrystalline phase at low temperatures which precedes further crystallization at much longer times, but above some temperature which varies for each alloy, most of the alloys crystallize in one rapid event. At all annealing temperatures, some fraction of each sample does not crystallize after the isothermal step, and this remaining amorphous phase has a higher average $T_{g}$ while also exhibiting reduced thermal stability. Equilibrium viscosity values determined for Vit1a-Vit1c, and thus kinetic properties in general, lie between those of Vit1 and Vit4. Differences in isothermal stability observed in low-temperature TTT diagrams have been attributed to a decomposition process which acts at low temperatures in most of the alloys in the series. This decomposition appears to be a precursor to primary crystallization of the quasicrystal phase. The isothermal stability in Vit1c and Vit4 is thus increased because of the reduced critical temperature and increased timescale of the decomposition process, though a complete explanation of this effect must also take into account the polymorphic nature of the crystallization process.

For complex multicomponent systems, it appears that commonly used kinetic and thermodynamic parameters fail to predict the GFA as accurately as they do in binary or ternary systems. Although all the studied alloys exhibit a relatively high viscosity, high thermal stability, and low critical cooling rate, the results detailed above reveal a contradictory trend. Those alloys which have the lowest critical cooling rate also have diminished thermal stability, despite the fact that they are the most viscous. This implies that knowledge of the complete TTT diagram is essential for comparing and predicting GFA among multicomponent bulk glass-forming systems.

\section{ACKNOWLEDGMENTS}

The authors acknolwedge the support of the National Aeronautics and Space Administration (Grant No. NAG81744) and the Department of Energy (Grant No. DEFG-0386ER45242). 
*Corresponding author. Electronic address: schroers@caltech.edu

${ }^{1}$ W. Clement, R. H. Willens, and P. Duwez, Nature (London) 187, 869 (1960).

${ }^{2}$ A. Inoue, T. Nakamura, N. Nishiyama, and T. Masumoto, Mater. Trans., JIM 33, 937 (1992).

${ }^{3}$ A. Inoue, T. Zhang, N. Nishiyama, K. Ohba, and T. Masumoto, Mater. Trans., JIM 34, 1234 (1993).

${ }^{4}$ A. Peker and W. L. Johnson, Appl. Phys. Lett. 63, 2342 (1993).

${ }^{5}$ N. Nishiyama and A. Inoue, Mater. Trans., JIM 37, 1531 (1996).

${ }^{6}$ A. L. Greer, Nature (London) 366, 303 (1993).

${ }^{7}$ R. Busch, W. Liu, and W. L. Johnson, J. Appl. Phys. 83, 4134 (1998).

${ }^{8}$ C. A. Angell, Science (Washington, DC, U.S.) 267, 1924 (1995).

${ }^{9}$ T. A. Waniuk, R. Busch, A. Masuhr, and W. L. Johnson, Acta Mater. 46, 5229 (1998).

${ }^{10}$ K. F. Kelton, Int. J. Non-Equilib. Process. 11, 141 (1998).

${ }^{11}$ A. Masuhr, R. Busch, and W. L. Johnson, Mater. Sci. Forum 269-272, 779 (1998).

${ }^{12}$ S. Schneider, P. Thiyagarajan, and W. L. Johnson, Appl. Phys. Lett. 68, 493 (1996).

${ }^{13}$ K. F. Kelton, Philos. Mag. Lett. 77, 337 (1998).

${ }^{14}$ H. Assadi and J. Schroers, Acta Mater. 50, 89 (2002).

${ }^{15}$ T. A. Waniuk, J. Schroers, and W. L. Johnson, Appl. Phys. Lett. 78, 1213 (2001).

${ }^{16}$ J. Schroers, A. Masuhr, R. Busch, and W. L. Johnson, Phys. Rev. B 60, 11855 (1999).
${ }^{17}$ N. Wanderka, M.-P. Macht, M. Seidel, S. Mechler, K. Stahl, and J. Z. Jiang, Appl. Phys. Lett. 77, 3935 (2000).

${ }^{18}$ Q. Wei, N. Wanderka, P. Schubert-Bischoff, M.-P. Macht, and S. Friedrich, J. Mater. Res. 15, 1729 (2000).

${ }^{19}$ E. Bakke, R. Busch, and W. L. Johnson, Mater. Sci. Forum 225, 95 (1996).

${ }^{20}$ M.-P. Macht, S. Mechler, M. Müller, and N. Wanderka, Mater. Sci. Forum 386-388, 99 (2002).

${ }^{21}$ R. Busch, E. Bakke, and W. L. Johnson, Acta Mater. 46, 4725 (1998).

${ }^{22}$ W. H. Wang, Q. Wei, S. Friedrich, M.-P. Macht, N. Wanderka, and H. Wollenberger, Appl. Phys. Lett. 71, 1053 (1997).

${ }^{23}$ J. Schroers, R. Busch, A. Masuhr, and W. L. Johnson, Appl. Phys. Lett. 74, 2806 (1999).

${ }^{24}$ J. F. Löffler, P. Thiyagarajan, and W. L. Johnson, J. Appl. Crystallogr. 33, 500 (2000).

${ }^{25}$ J. Schroers, R. Busch, and W. L. Johnson, Appl. Phys. Lett. 76, 2343 (2000).

${ }^{26}$ J. Schroers, D. Holland-Moritz, D. M. Herlach, and K. Urban, Phys. Rev. B 61, 14500 (2000).

${ }^{27}$ R. Busch, A. Masuhr, E. Bakke, and W. L. Johnson, in Structure and Dynamics of Glasses and Glass Formers, edited by C. A. Angel, K. L. Ngai, J. Kieffer, T. Egami, and G. U. Nienhaus, Mater. Res. Soc. Symp. Proc. No. 455 (Materials Research Society, Pittsburgh, 1997), p. 369. 